\title{
Statistical Properties of the Cluster Dynamics of the Systems of Statistical Mechanics
}

\author{
A. Gabrielov, V. Keilis-Borok $†$ Ya. Sinai $\ddagger$ and I. Zaliapin $\S$
}

\begin{abstract}
Cluster dynamics is the property of dynamics of systems of statistical mechanics when for given time the whole array of particles is decomposed onto finite clusters which move independently during a random interval of time. In this paper we study statistical properties and critical indices related to cluster dynamics.
\end{abstract}

Keywords: Boltzmann kinetic equation, Boltzmann-Grad limit transition, cluster dynamics, Maxwellian distribution.

\section{Introduction}

L. Boltzmann was the first who tried to explain the laws of thermodynamics and kinetics as corollaries of dynamics of large systems of interacting particles (see his classical book [Bol]). In particular, Boltzmann derived the

*Departments of Mathematics and Earth and Atmospheric Sciences, Purdue University, West Lafayette, IN 47907, USA

†Institute of Geophysics and Planetary Physics and Department of Earth and Space Sciences, University of California, Los Angeles, CA 90095, USA, and International Institute for Earthquake Prediction Theory and Mathematical Geophysics, Russian Academy of Sciences, Moscow, Russia

${ }_{\ddagger}^{\ddagger}$ Mathematics Department of Princeton University, Princeton, NJ 08544, USA and Landau Institute of Theoretical Physics, Moscow, Russia

$\S$ Department of Mathematics and Statistics, University of Nevada, Reno, NV 89557, USA 
classical Boltzmann kinetic equation based on his Stoßanzahlsatz which gave the first "dynamical" explanation of irreversibility. The Stoßanzahlsatz can be applied to any system of statistical mechanics which can be considered as a small perturbation of an ideal gas and undergoes the so-called BoltzmannGrad limit transition (see [L1]) when the length of the free path is of the order of the size of the whole system. O. Lanford (see [L1]) gave the first mathematical proof of the local existence theorem of the solutions of the Boltzmann equation where he showed the convergence of correlation functions to solution of the Boltzmann equation.

There were many attempts to extend the conditions under which the Boltzmann kinetic equation or its generalization works. In this connection we would like to mention the book by Bogolyubov [B1] where Bogolyubov stressed several times the idea that in the gaseous phase when the interaction between the particles is short-ranged the system can be decomposed onto finite clusters so that during some random interval of time each cluster moves independently on other clusters as a finite-dimensional dynamical system. After such random time the system can be decomposed again on other dynamically independent clusters and so on. It is natural to call this type of dynamics as cluster dynamics.

The cluster dynamics was shown to exist in the one-dimensional systems of statistical mechanics in [S1]. Consider the infinite system of onedimensional particles with pair-wise interaction having a hard core and short range. Assume that the distribution of particles is given by the Gibbs canonical distribution. In the one-dimensional case in the thermodynamical limit there is no difference between canonical and grand canonical distributions. According to these distributions in a typical situation on any interval $[-R, R]$ there are empty intervals of the length $O(\ln R)$ having at $t=0$ no particles. On the other hand, the velocities of the particles have Gaussian distributions. Therefore for any $T$ the velocities of the particles on $[-R, R]$ grow typically as $O(\sqrt{\ln R})$. It shows that for sufficiently large $R$ particles cannot go from 
one end-point of an empty interval to the other one. In other words for any $T$ in the finite-dimensional dynamics particles in the domains bounded by empty intervals do not interact with external particles.

This gives cluster dynamics. The accurate proof requires some probabilistic estimates which show that the velocities do not become large during the finite-dimensional dynamics (see [S1]). All these ideas were generalized to the multi-dimensional case and low density, i.e. to the gaseous phase in [S2].

The infinite system of equations of motion of particles $\left(x_{i}, v_{i}\right)$ of mass $m=1$ has the form

$$
\frac{d x_{i}}{d t}=v_{i}, \quad \frac{d v_{i}}{d t}=-\sum_{j} \frac{\partial U\left(\left|q_{i}(t)-q_{j}(t)\right|\right)}{\partial q_{i}}
$$

In the paper by E. Presutti, M. Pulvirenti and B. Tirozzi [PPT] the authors proved the general existence theorem for solutions of (1) for any limit Gibbs distribution with arbitrary density and inverse temperature. Their method is based upon the reduction of (1) to the corresponding integral equation and the proof of existence of solutions of the integral equation.

The most general results were obtained by R. Dobrushin and J. Fritz (see [DF1]). They described a large subset in the phase space of (1) for which they proved 1) the existence theorem of solutions of (1) and 2) the fact that the probability of this subset with respect to any natural Gibbs distribution is 1 . This gives the possibility to describe the dynamics in the space of Gibbs distributions.

Recently, it turned out that the concept of cluster dynamics has a wider domain of applications in such fields as plasma physics, geophysics and others (see [KS1, KS2, MDR+, RKB]). In this connection it is interesting to study the distributions of various characteristics of cluster dynamics, such as the distribution of the size of the clusters, the statistics of particles in a cluster and others. The whole set of problems resembles problems of percolation theory but seems to be more difficult than the percolation theory 
because it involves dynamical characteristics. There is no hope that something can be done analytically. On the other hand, the use of computers opens wide possibilities in getting numerical results. The corresponding experiments were performed recently and the results are presented below in this paper. We want to stress the appearance of new critical indices which are special for cluster dynamics and presumably are different from similar ones in percolation theory.

In this paper we analyze numerically cluster dynamics in a simplest system - a frictionless elastic billiard. The model and cluster rules are described in Sect. 2. We report a phase transition in the cluster formation process in Sect. 3 and describe how it depends on the model parameters in Sect. 4 . Also, we study how the size of the maximal cluster in a finite system scales with the system size (Sect. 5). Section 6 describes possible applications of cluster dynamics. Details of our numerical simulations are given in Sect. 7.

\section{Model}

The results in this paper refer to an elastic frictionless billiard on a square table. Namely, we consider $N$ balls of mass $m=1$ and radius $R$ placed within the region $\mathcal{T}=\{(x, y):|x| \leq L / 2,|y| \leq L / 2\}$. We will call $N$ the size of the billiard. Each ball moves without friction with constant velocity $\mathbf{v}_{i}=\left(v_{x}^{i}, v_{y}^{i}\right), i=1, \ldots, N$ until it collides with a wall or another ball. All collisions are elastic, which means that the total energy $E=\sum_{i=1}^{N} m\left|\mathbf{v}_{i}\right|^{2} / 2$, $\left|\mathbf{v}_{i}\right|^{2}=\left(v_{x}^{i}\right)^{2}+\left(v_{y}^{i}\right)^{2}$, of the system remains constant, the total momentum

$\mathbf{p}=\sum_{i=1}^{N} m \mathbf{v}_{i}$ is not affected by ball collisions, and in wall collisions the ball's reflection and incidence angles are the same. A useful characteristic of the billiard is the density of balls:

$$
\rho=\frac{N \pi R^{2}}{L^{2}}
$$

We will need the notion of $\Delta$-cluster [S1], which is a group of balls that have affected each others' dynamics during the time interval of duration $\Delta$. 
Formally, we call two balls $\Delta$-neighbors at epoch $t$ if they collided during the time interval $[t-\Delta, t]$. Any connected component of this neighbour relation is called a $\Delta$-cluster at epoch $t$. This definition ensures that each ball has collided with at least one ball from its $\Delta$-cluster within the time interval $[t-\Delta, t]$. The mass of a cluster is the total mass of its balls. We denote by $N_{\Delta}(t)$ the total number of $\Delta$-clusters at instant $t$, and by $M_{\Delta}^{i}(t)$ the mass of the $i$-th largest cluster. Thus, the mass of the maximal $\Delta$-cluster is $M_{\Delta}^{1}(t)$, of the second largest cluster $M_{\Delta}^{2}(t)$, of the smallest cluster $M_{\Delta}^{N_{\Delta}}(t)$, etc.

Obviously, at $\Delta=0$ there exist $N$ clusters of mass $m$, each corresponding to an individual ball. As $\Delta$ increases, the balls start to collide so the number of clusters becomes smaller while their masses increase. In this note we only consider a situation when $\Delta=t$, that is we deal with all the clusters that have been formed during the time interval $[0, \Delta]$. This allows us to drop the dependence on $t$ and work with the number of clusters $N_{\Delta}$, maximal cluster of mass $M_{\Delta}^{1}$, etc.

In this paper we focus on the time-dependent distribution (density) $f_{t}(M)$ of cluster sizes (masses). In a numerical study, to obtain a sample of $n$ clusters at epoch $t$ one runs the model $n$ times, each time choosing a single cluster at epoch $t$. A standard practice in stochastic geometry suggests to choose the cluster nearest to the origin (that is the cluster that contains the ball nearest to the origin). It happens that the computational load associated with this procedure is prohibitive for our purposes. To make the computations workable, we approximate the cluster size distribution $f_{t}(M)$ using the entire cluster population from a single realization of the model, which has density $g_{t}(M)$. For large $N, f_{t}(M)$ becomes an area-biased version of $g_{t}(M)$, since large clusters have a better chance to lie closer to the origin than smaller ones; and the two distributions are connected via [SKM]:

$$
f_{t}(M)=\frac{M g_{t}(M)}{\int M g_{t}(M) d M} .
$$

The results in this paper refer to $g_{t}(M)$. 


\section{Phase transition in dynamical clustering}

To study the dynamics of clusters we have considered models with $10^{-6} \leq$ $\rho \leq 10^{-1}$, and $10 \leq N \leq 10^{4}$. We run each model until the instant when a cluster of mass $0.95 \times N$ is formed.

Figure 1 shows fractional masses $\mu_{\Delta}^{i}=M_{\Delta}^{i} / N$ of clusters as a function of time when they have been formed; this figure refers to $\rho=10^{-3}, N=10^{4}$. Here we see the following generic qualitative picture that has been observed for all the models considered. At $\Delta=0$, we start with $N$ clusters of mass $m$. As the model evolves, clusters of increasingly larger masses are formed and the total number of clusters decreases. For some period of time, $0 \leq t \leq t_{c}$ (for this model, $t_{c} \approx 510$ ) the cluster mass distribution has no notable gaps, in particular the size of the largest cluster does not exceed significantly the size of the second largest cluster. At $t=t_{c}$ one observes a sharp qualitative change in the cluster formation process. There appears a distinctive largest cluster, which creates a gap in the mass distribution between the largest cluster and the rest of the clusters. Notably, this change happens when the largest cluster is still relatively small: less than $10 \%$ of the total system mass.

It will be convenient to define formally the time instant $t_{c}$ that corresponds to the change in cluster formation. We define here $t_{c}$ as the instant after which there are no clusters larger than $M_{t_{c}}^{1}$ :

$$
t_{c}=\inf \left\{t: M_{t}^{1}>M_{s}^{i}, s>t, i>1\right\} .
$$

We will refer to $t_{c}$ as critical time. Note that this definition uses the information from the times $t>t_{c}$ (i.e., $t_{c}$ is not a stopping time), so it cannot be used for operational detection of $t_{c}$. It would be useful to have an alternative definition which will use only the information available prior $t_{c}$.

Figure 2 shows the empirical cluster mass density for the model with $\rho=10^{-3}$ and $N=10^{4}$ at three time instants depicted by vertical lines in Fig. 1. For $t \leq t_{c}$ the cluster mass distribution $g_{t}(M)$ is well described by a 
power law with an exponential taper:

$$
g_{t}(M)=M^{-\beta} \exp \left(-\frac{M}{\gamma(t)}\right) .
$$

Here $\beta \approx 5 / 2$, and the taper strength is determined by $\gamma(t): \gamma(t) \ll M$ leads to almost an exponential distribution, while $\gamma(t) \gg M$ to a pure power law. Our simulations suggest that $\gamma(t)$ is monotone increasing with $\gamma\left(t_{c}\right)=\infty$, so that $g_{t}(M)$ transforms from exponential at small times $t \ll t_{c}$ to a pure power law at the critical epoch $t_{c}$. One can readily see the scenario that characterizes systems with phase transition of second kind. In statistical mechanics, equation (5) describes the correlation function $\Gamma(r)$ of a system (e.g., Potts model) near the critical point. A similar power law with exponential taper was sugegsted for the mass distribution of clusters in a percolation model $[\mathrm{SA}, \mathrm{MNS}+]$ as the percolation instant approaches; for rupture sizes in a colliding cascades model of earthquakes [GKZ+1, GKZ+2, ZKG]; for real ruptures in steel samples $[\mathrm{RKB}]$, etc. For $t>t_{c}$ the distribution is decomposed into two components: A $\delta$-function at the mass of the largest cluster, and the tapered power law (5) for the rest of the clusters with $\gamma(t)$ monotone decreasing for $t>t_{c}$.

This picture is observed for all the models considered independently of the billiard density $\rho$ and size $N$. In particular, the critical index $\beta \approx 5 / 2$ is universal: Figure 3 show the cluster mass distribution at $t_{c}$ for seven different models with $N=5 \times 10^{3}$ and $\rho=10^{-1}, 10^{-2}, \ldots, 10^{-7}$.

\section{Scaling of critical time and mass}

Here we study how the critical instant $t_{c}$ and the fractional mass $\mu_{c}:=$ $M_{t_{c}}^{1} / N$ of the largest cluster at $t_{c}$ depend on the billiard density $\rho$. For that we run multiple (from 10 to 1000) realizations of the billiard for $\rho=$ $10^{-1}, 10^{-2}, \ldots, 10^{-7}$ and $N=5 \times 10^{3}$. The averaged values of $t_{c}$ and $\mu_{c}$ are shown in Figs. 4, 5 as functions of $\rho$. These results support the following 
asymptotic relations, which give very good approximation for $\rho<10^{-2}$ :

$$
t_{c}\left(\rho \mid N=5 \times 10^{3}\right) \approx 0.4 \rho^{-1}, \quad \mu_{c}\left(\rho \mid N=5 \times 10^{3}\right) \approx 0.07, \quad \text { as } \rho \rightarrow 0 .
$$

A more detailed analysis (not shown) indicates that the large density correction for critical time is given by:

$$
t_{c}\left(\rho \mid N=5 \times 10^{3}\right)=0.4 \rho^{-1}\left(1+\rho^{0.4}+o\left(\rho^{0.4}\right)\right), \rho \rightarrow 0 .
$$

\section{Size of the maximal cluster}

Here we consider the dynamics and scaling of the maximal cluster. Figure 6 shows the dynamics of the fractional mass $\mu_{\Delta}^{1}=M_{\Delta}^{1} / N$ of the largest cluster averaged over 10 to 1000 realizations of a billiard; it refers to ten models with $10 \leq N \leq 10^{4}$ and fixed $\rho=10^{-3}$. By construction, we have the following limiting relations:

$$
M_{0}^{1}=1, \quad M_{\infty}^{1}=N .
$$

Next we study in detail the scaling of $M_{\Delta}^{1}$ as a function of $N$ and $\Delta$. Figure 7 shows $M_{\Delta}^{1}$ as a function of $N$ at eight time instants between $\Delta=0$ and $\Delta=1400 \approx 3 \times t_{c}$. One can see a gradual transition between the limiting scalings (8). Approximately linear form of the plots suggests that we have a power law dependence

$$
M_{\Delta}^{1}=c(\Delta) N^{\alpha(\Delta)}
$$

with $c(0)=c(\infty)=1, \alpha(0)=0$, and $\alpha(\infty)=1$. Maximum likelihood estimations of $c(\Delta)$ and $\alpha(\Delta)$ for $0 \leq \Delta \leq 1400$ are shown in Fig. 8. At critical time we have $\alpha\left(t_{c}\right) \approx 2 / 3$.

A more detailed analysis (not shown) indicates that at initial times $(0<$ $\left.t<t_{c} / 3\right)$ the maximal cluster size in a finite syztem of size $N$ is proportional to $\log _{10} N$ :

$$
M_{\Delta}^{1}=\alpha^{\prime}(\Delta) \log _{10}(N)+c^{\prime}(\Delta)
$$

There exists a natural connection between this transition from logarithmic to power scaling of the maximal cluster size and from exponential to 
power tail of the cluster size distribution (see Eq. (5) and Fig. 2). Indeed, suppose that cluster sizes are independent and identically distributed with distribution function $F(x)$ and density $f(x)$. Then the size of the maximal among $N$ clusters has distribution $F_{\max }(M)=F^{N}(M)$ and density $f_{\max }(M)=N f F^{N-1}$; and the most probable size $M^{*}$ of the maximal cluster is given by the finite solution of $\frac{d}{d M} f_{\max }\left(M^{*}\right)=0$. It is readily verified that

$$
\begin{gathered}
F(M)=1-e^{-\lambda M} \Rightarrow M^{*}=\frac{\ln (N)}{\lambda} \propto \ln (N), \\
F(M)=1-M^{-b} \Rightarrow M^{*}=\left(\frac{N b+1}{b+1}\right)^{1 / b} \propto N^{1 / b}+o\left(N^{1 / b}\right) .
\end{gathered}
$$

The exponential tail of the cluster size distribution at $t<t_{c}$ thus justifies the logarithmic dependence (10) of the maximal cluster size on $N$; while the power-law cluster size distribution around $t_{c}$ explains the power-law scaling (9). Equations (9) and (12) suggest $b=1 / \alpha$. We notice that the index $b=\beta-1 \approx 3 / 2$ of the cluster size distribution observed at $t_{c}$ (see Fig. 3 ) is indeed equal to $1 / \alpha \approx 1 / 0.66 \approx 3 / 2$ observed at $t_{c} \approx 510$ in the top panel of Fig. 8 .

\section{Possible applications of cluster dynamics}

We have demonstrated in Sect. 3 (Figs. 1,2) above that the distribution of the cluster size in the frictionless elastic billiard changes as the phase transition approaches. A similar phenomenon has been observed in seismicity and economics, where it is related to the approach of an appropriately defined critical event. Below we briefly discuss cluster dynamics in seismicity. More detailed discussion and further examples, suggesting universality of that phenomenon, are given in [KSS+, KS1, KS2].

Seismicity. An earthquake is an episode of rupture and discontinuous displacement in the outer shell of solid Earth, called the lithosphere. Generally speaking, nucleation of a single earthquake is controlled by the clash between 
stress- and strength-fields in its vicinity. Both these fields, particularly the strength, are in turn controlled by a multitude of processes generating strong instability. Among them are interactions with other earthquakes, mechanical and chemical interactions of rocks and fluids, phase transitions of minerals, heat flow, non-linear deformations and fracturing, interactions between geospheres, incompatibility between the structure and kinematics, etc. These processes evolve in multiple scales, from global to microscopic ones. Altogether they turn the lithosphere into a hierarchical dissipative non-linear ("complex") system. Fundamental equations connecting seismicity with this set of intertwined mechanisms are not yet known.

Scale invariance. Size of an earthquake is usually defined as its magnitude $m$ - a logarithmic measure of the energy $E$ released by the earthquake [Kan] $\left(m=2 / 3 \log _{10} E+C\right)$. About $10^{6}$ earthquakes with $m>2$ are recorded annually worldwide, and once in a few years the largest earthquakes with $m>8$ occur. The magnitude distribution of earthquakes is known in seismology as the Gutenberg-Richter law [GR1, GR2]:

$$
\log _{10}(N(m))=a-b m
$$

Here $N(m)$ is the average annual number of earthquakes with magnitude $m$ or more, $b \approx 1$. The Gutenberg-Richter law emerges only after considerable averaging of seismicity over time and territory and gives a good description for small to medium magnitudes. At the relatively large magnitudes, the size distribution bends downwards. The linear relation (13) is equivalent to the power law distribution of earthquake energy:

$$
N(E) \propto E^{-\beta}, \quad \beta \approx 2 / 3
$$

To accommodate the downward slope of the energy distribution at larger magnitudes, it can be approximated by an exponentially tapered distribution:

$$
N(E) \propto E^{-\beta} \exp \left(E / E_{0}\right)
$$


Strong earthquakes are usually defined as outliers in observed distributions — "extreme events". Such earthquakes are associated with abrupt overall changes of seismicity, and, qualitatively, with phase transitions.

Clustering and phase transitions. Earthquakes come in a hierarchy of clusters formed in a broad range of time-, space-, and magnitude scales, from microscopic to global ones. Clusters emerge, coalesce, split, migrate, and alternate with seismic quiescence ("anti-clusters"). As a strong earthquake approaches, distribution of cluster sizes tends to change in favor of larger clusters. Similarly, the magnitude distribution of individual earthquakes changes in favor of larger magnitudes. In particular, the distribution (15) changes similarly to the cluster size distribution (5) described in Sect. 3. These phenomena have been found in real seismicity of numerous regions worldwide as well as in numerical models of seismicity. They were used in several earthquake prediction algorithms, self-adjusting to statistical properties of seismicity in different regions [KS2]. Statistical significance of predictions based on that kind of clusters is demonstrated in [MDR+].

Similar changes in scaling relations have been observed before American economic recessions and some socio-economic extreme events [KS1].

\section{$7 \quad$ Parameters and simulation details}

We have simulated the billiard for $1 \leq N \leq 10^{4}$ and $10^{-8} \leq \rho \leq 1 / 2$. The table size $L$ was kept constant, while appropriate values of $R$ for any given $\rho$ and $N$ were calculated via (2). We put $m=1$ and $T=1 / k_{B}$.

Recall that the Maxwellian velocity distribution of an ideal gas in $D$ dimensional space is given by

$$
f(\mathbf{v})=\left(\frac{m}{2 \pi k_{B} T}\right)^{D / 2} \exp ^{-m|\mathbf{v}|^{2} /\left(2 k_{B} T\right)},
$$

where $\mathbf{v}=\left(v_{1}, \ldots, v_{D}\right),|\mathbf{v}|^{2}=v_{1}^{2}+\cdots+v_{D}^{2}, k_{B}$ is Boltzmann constant, $T$ temperature, and $m$ particle mass. Accordingly, the speed $s:=|\mathbf{v}|$ of 
particles in a $2 D$ system is given by Chi-distribution with two degrees of freedom:

$$
p(s)=s \sqrt{\frac{m}{k_{B} T}} \exp ^{-m s^{2} /\left(2 k_{B} T\right)} .
$$

We have verified that any (non-degenerate) initial velocity distribution eventually transforms to the Maxwellian distribution (16). The rate of convergence is rather fast; say, the isotropic uniform initial velocity distribution for $N=500$ balls transforms into the Maxwellian distribution after 2000 ball collisions, which is 4 collisions per ball on average.

We start all our models with the Maxwellian velocity distribution (16) and uniform placement of the balls. Specifically, the balls are placed on the table one-by-one. The center of the first ball is uniformly distributed within the region

$$
\mathcal{T}^{\prime}=\{(x, y):|x|<L / 2-R,|y|<L / 2-R\},
$$

which ensures that it does not intersects with the table walls. The center of $(k+1)$-th ball, $k \geq 1$, is uniformly distributed within the region $\mathcal{T}^{\prime} \backslash B_{k}$, where $B_{k}$ is the union of already placed balls; this ensures the new ball does not intersects with existing balls and the walls.

Acknowledgment. This study was partly supported by NSF grants ATM 0327558 and ATM 0327598.

\section{References}

[Bol] L. Boltzmann Vorlesungen über Gastheorie. Bands I, II, 1896.

[B1] N. Bogolyubov Problems of Dynamical Theory in Statistical Phyics. GITTL, Moscow-Leningrad, 1946.

[L1] O. Lanford III Time Evolution of Large Classical Systems. Lecture Notes in Physics, N38, 1975. 
[S1] Ya.G. Sinai Construction of Cluster Dynamics for Dynamical Systems of Statistical Mechanics. Proc. of Moscow State University, N1, 152, 1974.

[S2] Ya.G. Sinai Constuction of Dynmaics in Infinite Systems of Particles. Theoretical and Mathematical Physics, 12, 487, 1973.

[PPT] E. Presutti, M. Pulvirenti, B. Tirozzi Time Evolution of Infinite Classical Systems with Singular, Long Range, Two-Body Interactions. Comm. in Math. Physics, 47, 81-95, 1976.

[DF1] R. Dobrushin, J. Fritz Non-equilibrium Dynamics of One-dimensional Infinite Systems with a Hard-Core Interaction. Comm. in Math. Physics, 55, 275-292, 1977.

[Kan] Kanamori, H. The energy release in great earthquakes, J. Geophys. Res., 82, 2981-2987, 1977.

[GR1] Gutenberg, B., and C. F. Richter Seismicity of the Earth, Geol. Soc. Amer., Special papers, 34, 1-131, 1941.

[GR2] Gutenberg, B., and C. F. Richter Frequency of earthquakes in California, Bull. Seism. Soc. Am., 34, 185-188, 1944.

[SKM] D. Stoyan, W. S. Kendall, and J. Mecke Stochastic Geometry and Its Applications, 2nd ed. New York: Wiley, 1987.

[SA] Stauffer, D., Aharony, A. Introduction to percolation theory. 2-nd ed., Taylor \& Francis, 1994.

[MNS+] Margolina, A., Nakanishi, H., Stauffer, D., and Stanley, H. E. Monte Carlo and series study of corrections to scaling in two-dimensional percolation. J. Phys. A, 17, 1683-1701, 1984.

[GKZ+1] Gabrielov, A. M., Keilis-Borok, V. I., Zaliapin I. V., and Newman W. I. Critical transitions in colliding cascades. Phys. Rev. E, 62, 237-249, 2000. 
[GKZ+2] Gabrielov, A. M., Zaliapin I. V., Keilis-Borok, V. I., and Newman W. I. Colliding Cascades as a Model for Earthquake Prediction. Geophys. J. Int., 143, 427-437, 2000.

[RKB] Rotwain, I., Keilis-Borok V., Botvina, L. Premonitory transformation of steel fracturing and seismicity. Phys. Earth Planet. Inter., 101, 61-71, 1997.

[ZKG] Zaliapin, I., V. Keilis-Borok and M. Ghil A Boolean Delay Model of Colliding Cascades. II: Prediction of Critical Transitions. J. Stat. Phys., 111, 3-4, 839-861, 2003.

[ZWG] Zaliapin, I., H. Wong and A. Gabrielov Inverse cascade in percolation model: Hierarchical description of time-dependent scaling. Phys. Rev. E, 71, 066118, 2005.

[KSS+] Keilis-Borok, V., J. H. Stock, A. Soloviev, and P. Mikhalev Prerecession pattern of six economic indicators in the USA. J. Forecasting, 19, 65-80, 2000.

[KS1] Keilis-Borok,V., and A. Soloviev On universal precursors to extreme socio-economic events. Geophysical Research Abstracts, Volume 7, 2005. Proc. of the EGU General Assembly 2006, Vienna, Austria, 2-7 April 2006 (CD-ROM): EGU06-A-01498, 2005.

[KS2] Keilis-Borok, V. I., and Soloviev, A. A. (eds.) Nonlinear Dynamics of the Lithosphere and Earthquake Prediction, Springer-Verlag, Heidelberg, 337 pp., 2003.

[SKG+] Shebalin, P., V. Keilis-Borok, A. Gabrielov, I. Zaliapin and D. Turcotte Short-term earthquake prediction by reverse analysis of lithosphere dynamics. Tectonophysics, 413(1-2), 63-75, 2006. doi:10.1016/j.tecto.2005.10.033 
[MDR+] G. M. Molchan, O. E. Dmitrieva, I. M. Rotwain and J. Dewey Statistical analysis of the results of earthquake prediction, based on bursts of aftershocks. Phys. Earth Planet. Inter., 61, 128-139, 1990. 


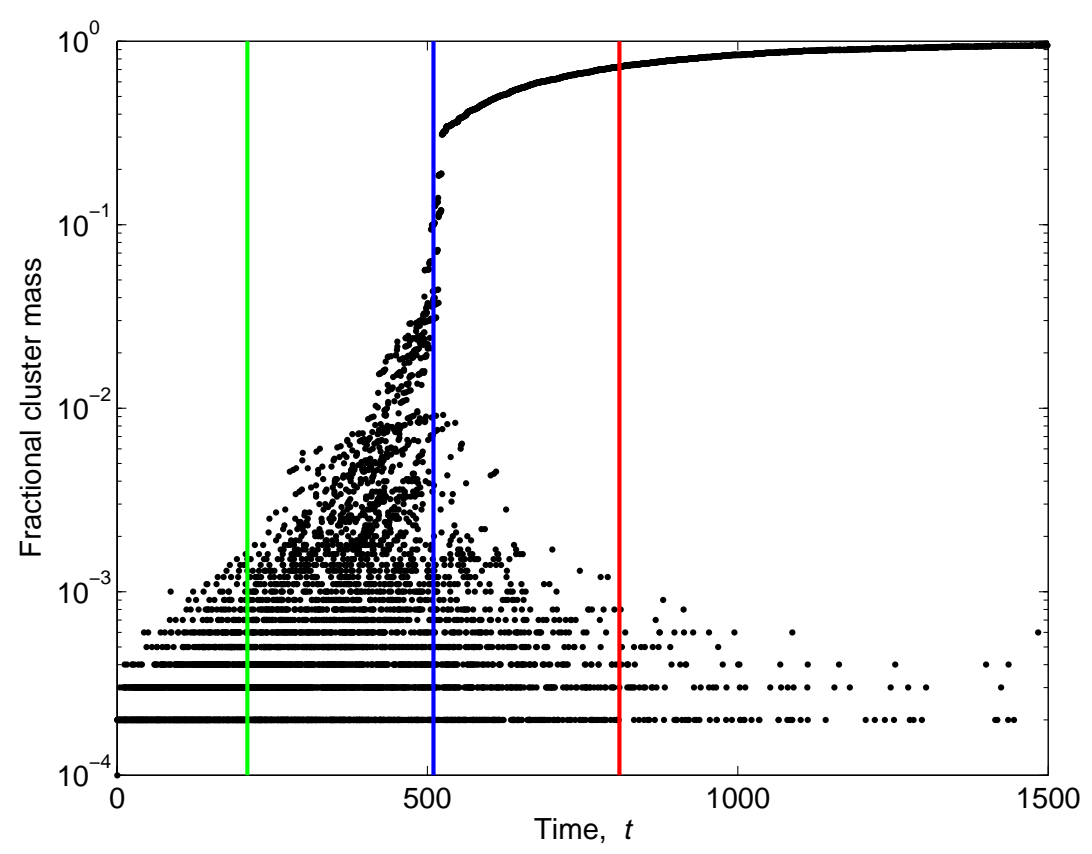

Figure 1: Fractional cluster masses $\mu_{\Delta}^{i}=M_{\Delta}^{i} / N$ as a function of time when they have been formed in a model with $\rho=10^{-3}, N=10^{4}$. Each point corresponds to a single cluster. The horizontal lines at the lower part of the figure are formed by a multitude of clusters of mass $M=2$ (lowest line), $M=3$ (second line), etc. The point $\left(0,10^{-4}\right)$ refers to $N=10^{4}$ balls, each of which forms a cluster of unit mass at $t=0$. Notice the dramatic change in the cluster mass distribution at the moment $t_{c} \approx 510$ depicted by blue vertical line. Three vertical lines correspond to the three cluster size distributions in Fig.2. 


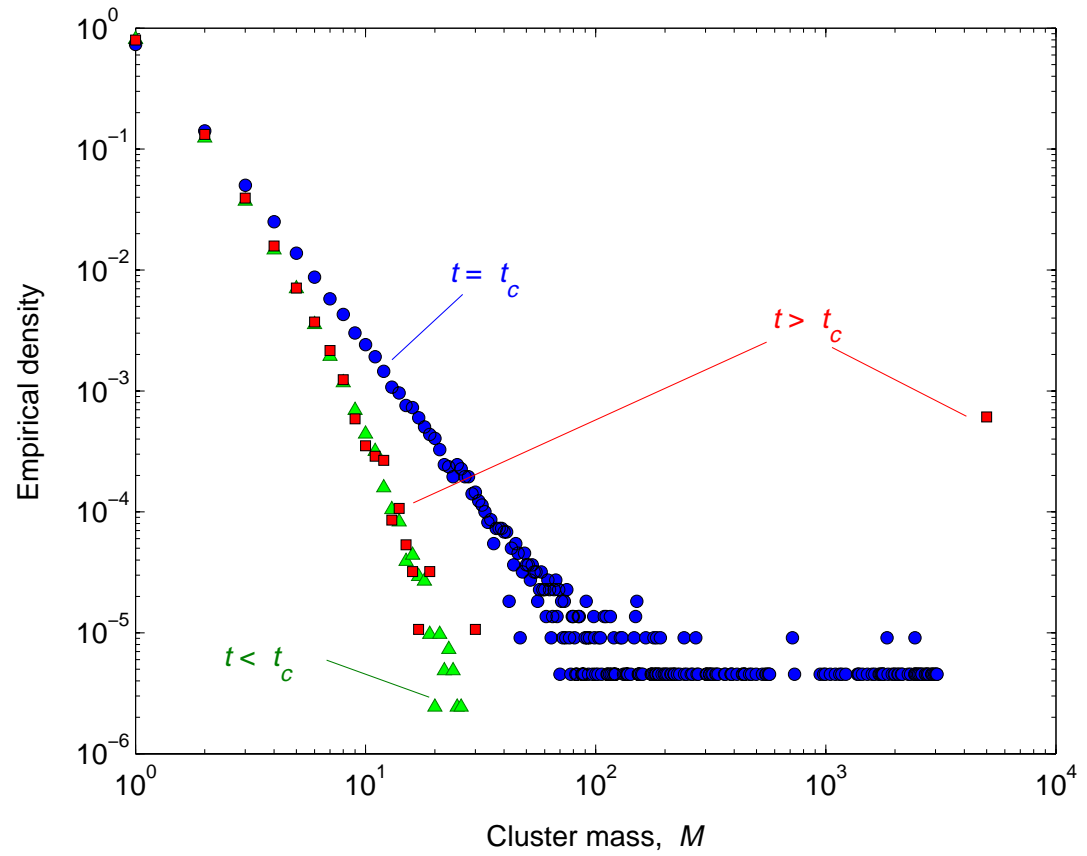

Figure 2: Cluster size distribution at three instants depicted by vertical lines in Fig. 1. At $t<t_{c}$ (green triangles) distribution can be approximated by a power law with exponential taper at the tail; at $t \approx t_{c}$ (blue balls) it is a pure power law; at $t>t_{c}$ (red squares) it is a tapered power law plus a $\delta$ function at the largest cluster. To produce this figure we used 50 independent realizations of the model with $\rho=10^{-3}, N=10^{4}$. 


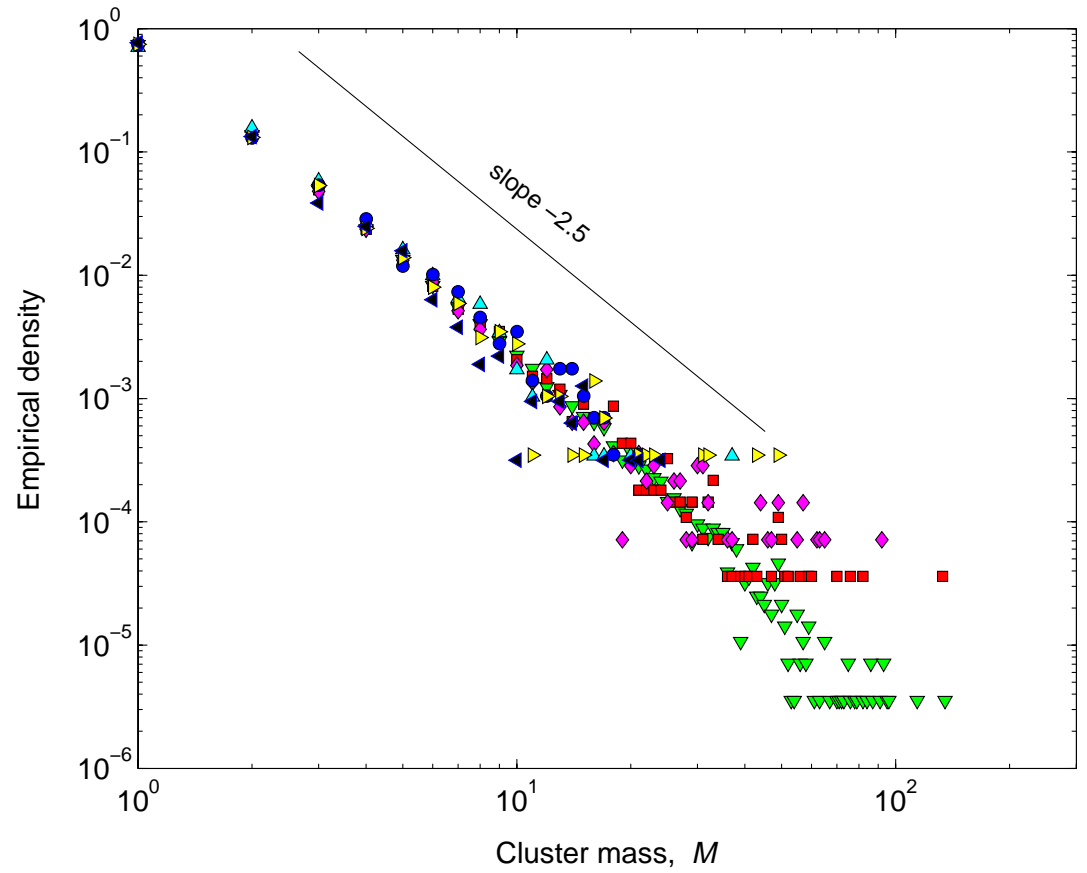

Figure 3: Power-law cluster size distribution at critical instant $t_{c}$ for seven models with fixed $N=5 \times 10^{3}$ and $\rho=10^{-1}, 10^{-2}, \ldots, 10^{-7}$. The distribution at critical instant $t_{c}$ is characterized by a universal index $\beta=5 / 2$.

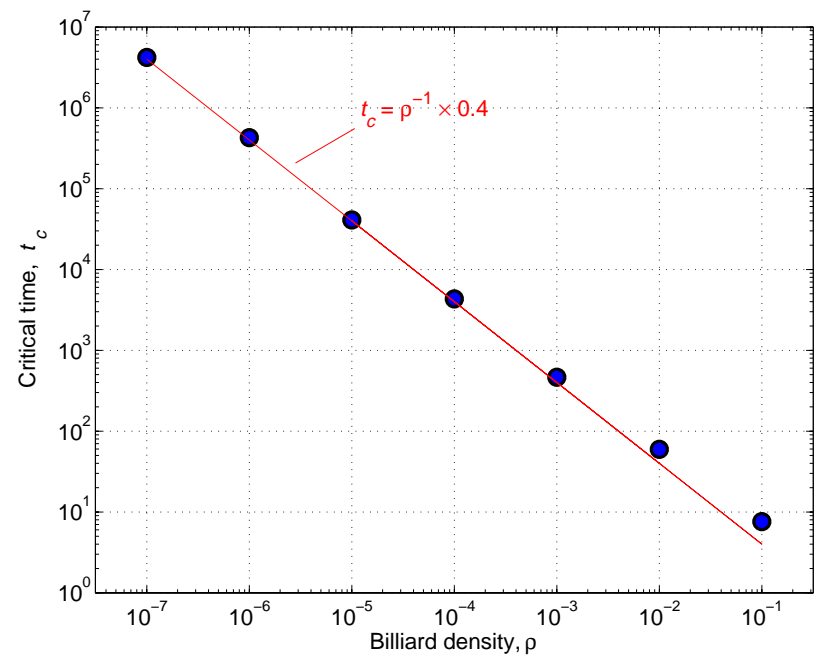

Figure 4: Scaling of critical time $t_{c}$ with the billiard density $\rho$ at fixed $N=$ $5 \times 10^{3}$. 


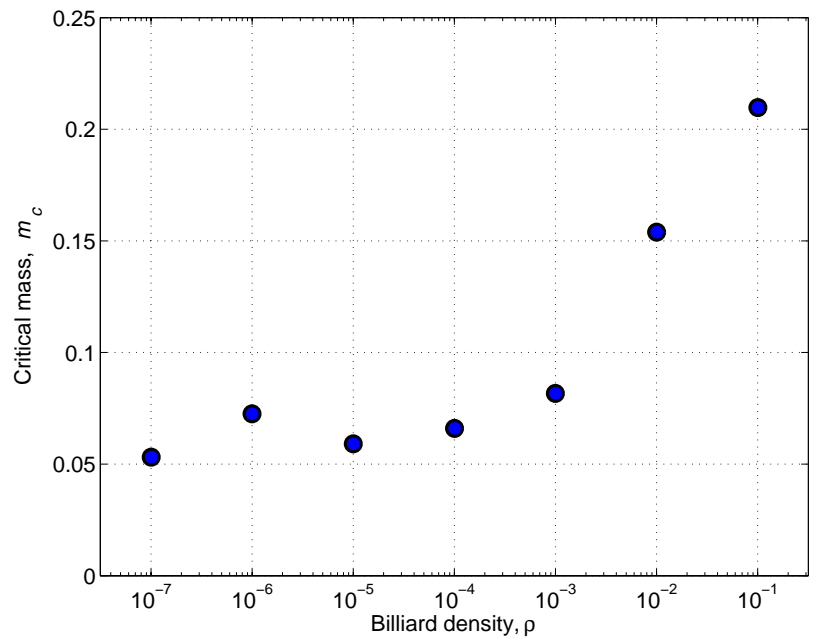

Figure 5: Critical mass $\mu_{c}$ as a function of the billiard density $\rho$ at fixed $N=5 \times 10^{3}$.

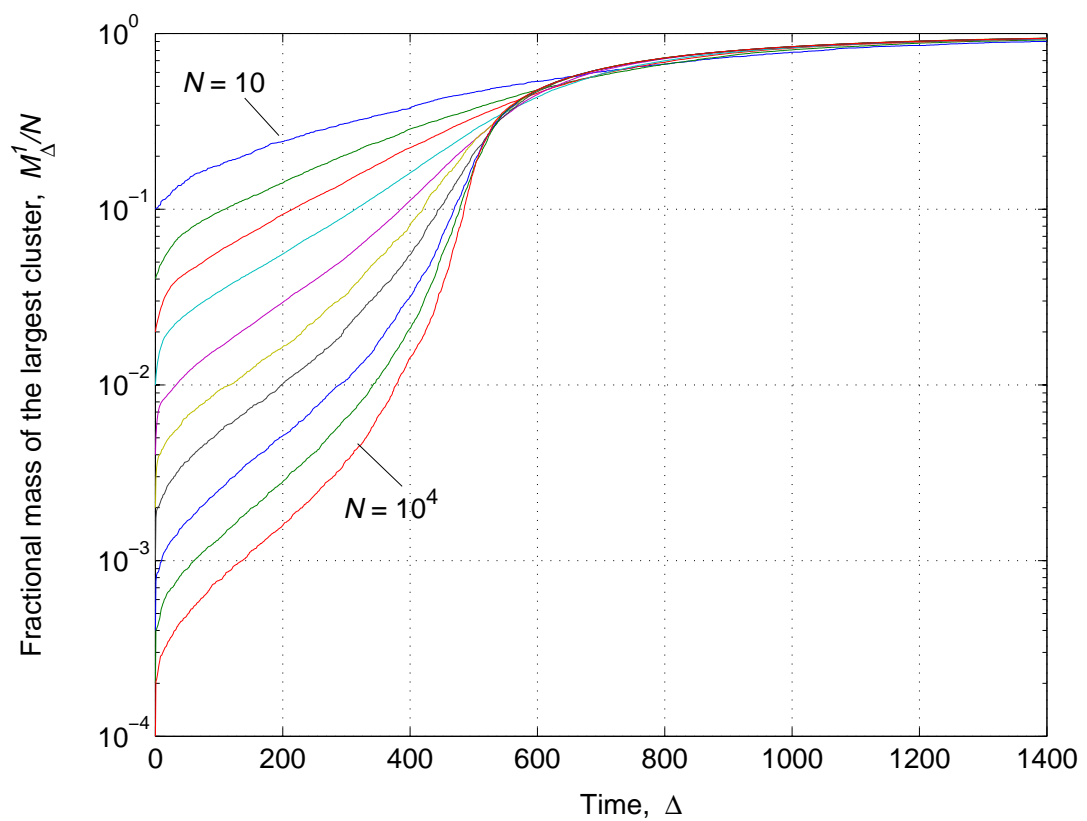

Figure 6: Fractional mass $\mu_{\Delta}^{1}=M_{\Delta}^{1} / N$ of the maximal cluster as a function of $\Delta$ for ten models with fixed $\rho=10^{-3}$ and $10 \leq N \leq 10^{4}$ (each line corresponds to a distinct value of $N$ ). 


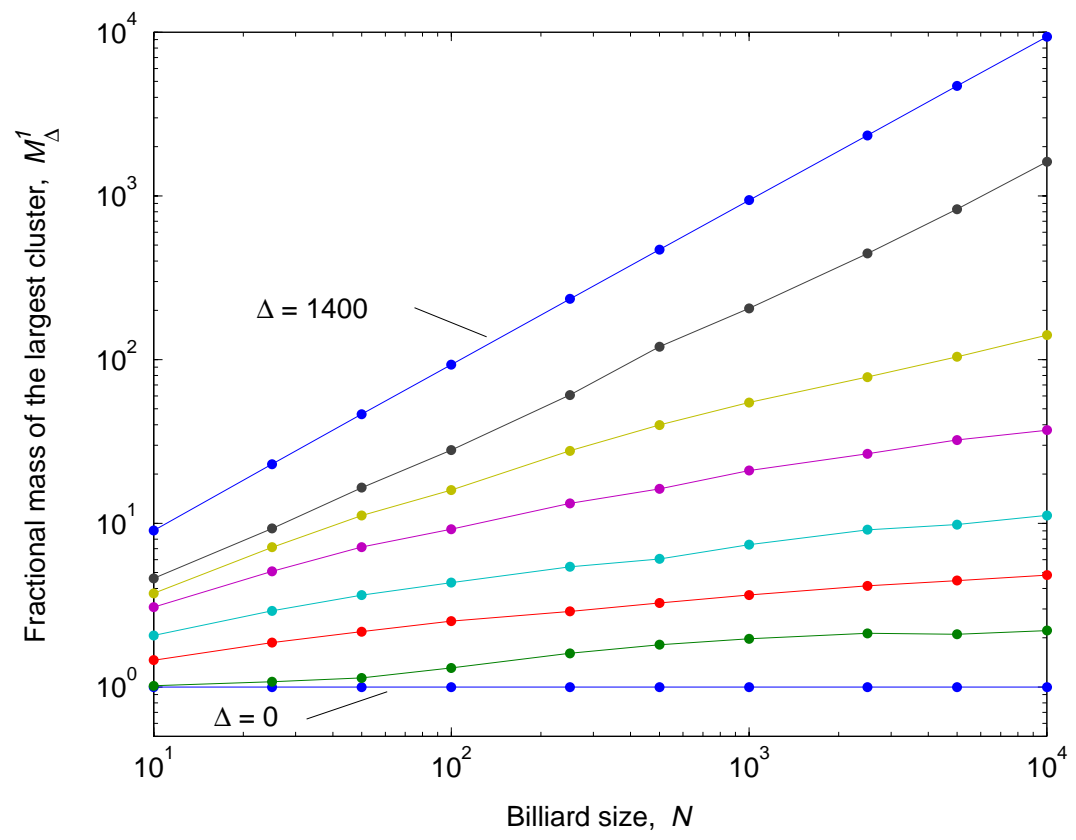

Figure 7: Mass $M_{\Delta}^{1}$ of the maximal cluster as a function of billiard size $N$ - each line corresponds to a distinct value of $\Delta$. Experiment is done under fixed $\rho=10^{-3}$.
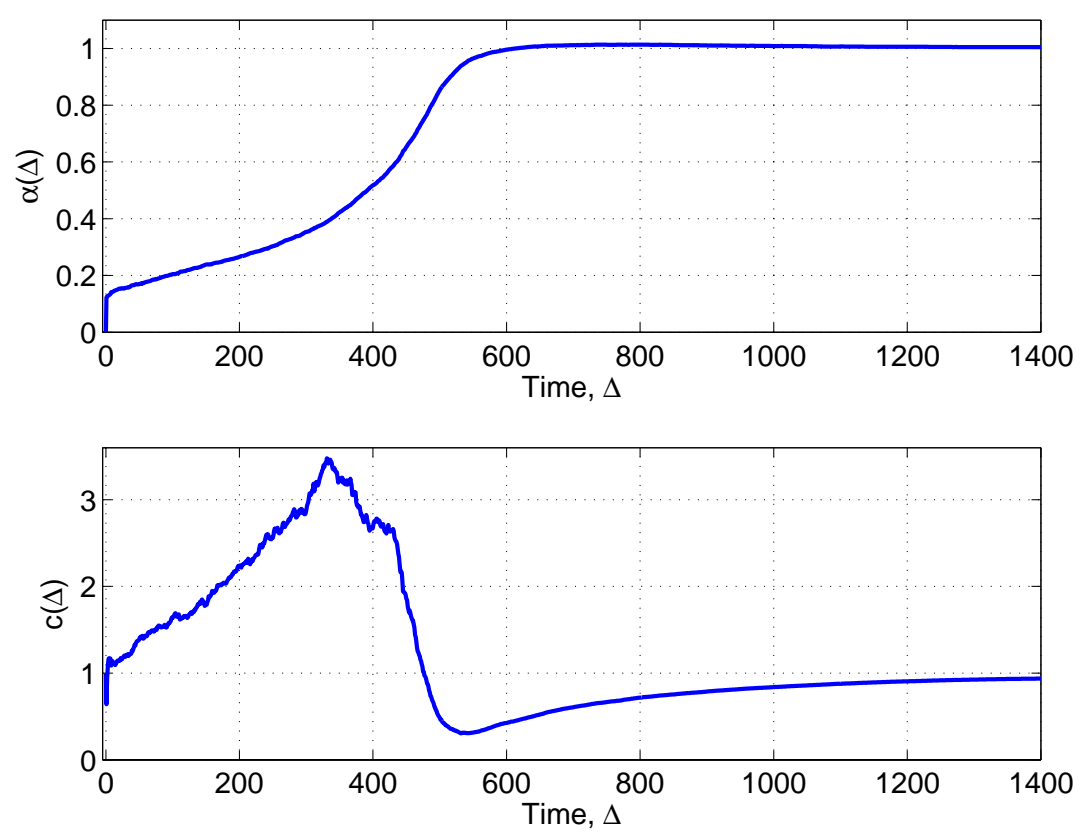

Figure 8: Parameters $\alpha$ and $c$ of scaling law (9) as functions of time $\Delta$. Each line in Fig. 7 corresponds to one point in each panel of this figure. To produce this figure we used models with fixed $\rho=10^{-3}$, and $10 \leq N \leq 10^{4}$. 